\title{
Easiness of sperms retrieval in non-obstructive azoospermia is a strong predictor of clinical pregnancy after intracytoplasmic sperm injection
}

\author{
Original \\ Medhat Kamel Amer', Ahmad Raef Sadek', Wael Zohdy', Sameh Fayek Gamal El Din', \\ Article \\ Ahmed Mostafa Abd El Baseer ${ }^{3}$, Ahmed Ragab Ahmed ${ }^{2}$ \\ ${ }^{1}$ Department of Andrology, Sexology and STDs, Kasr Al-Ainy Faculty of Medicine, Cairo \\ University, Cairo, ${ }^{2}$ Beni-Suef Faculty of Medicine, Beni-Suef University, Beni-Suef, ${ }^{3}$ Ministry \\ of Health and Population, Cairo, Egypt.
}

\begin{abstract}
Background: Testicular sperm extraction followed by Intracytoplasmic Sperm Injection (TESE-ICSI) is the only route to genetically father a child in couples with Non-Obstructive Azoospermia (NOA).

Objective: The aim was to identify the effect of severity of NOA on clinical pregnancy following ICSI.

Patients and Methods: This retrospective study included 643 patients who underwent 712 ICSI cycles at a specialized InVitro Fertilization center between 2014 and 2019. We classified the patients into easy (445) and difficult NOA (267) based on the difficulty of microdissection TESE procedure, number of sperm retrieved, and the number of surgical attempts.

Results: The clinical pregnancy rate is significantly higher in the easy NOA group [167/299 (55.8\%)] as compared with the difficult NOA group [68/194 (35.1\%)]. The mean age of the females and the mean follicle stimulating hormone (FSH) levels were significantly lower in the easy NOA group compared with the difficult group [27.5 \pm 4.3 vs $29.9 \pm 4.6$ years, $P=0.0001$, and $6.8 \pm 2.1$ vs $7.4 \pm 2.5 \mathrm{mIU} / \mathrm{ml}, P=0.004$, respectively]. There were no significant differences between the 2 groups regarding the mean male age, FSH level, and testicular size. The cumulus mass and the number of mature oocytes injected correlated significantly with the clinical pregnancy $(r=0.122, P=0.012$ and $r=0.145, P=0.003$, respectively). The sperm source whether fresh or frozen, the testicular pathology, and the identification of round spermatids in the pathology specimens did not correlate with the clinical pregnancy. We used the binary logistic regression to identify the final predictors. Only the NOA severity could be used to predict ICSI outcome. Easy NOA is more likely to have positive outcomes with ICSI (Odds ratio=1.70; Confidence interval=1.069-2.71; $P=0.025$ ). The model showed that neither female age nor FSH level could predict ICSI outcome in those patients.

Conclusion: Easy sperm retrieval is an independent factor that is associated with positive ICSI outcomes in couples with NOA.
\end{abstract}

Key Words: Difficult NOA, easy NOA, ICSI outcome, micro-TESE.

Received: 14 June 2020, Accepted: 05 July 2020.

Corresponding Author: Ahmed Ragab Ahmed, Department of Andrology, Sexology and STDs, Faculty of Medicine, Beni-Suef University, Beni- Suef, Egypt Tel.: 01009800611, E-mail: drahmedragab1981@gmail.com

ISSN: 2090-6048, 2020

\section{INTRODUCTION}

Infertility was ranked by the WHO (2011) as one of the major health problems associated with disability among people younger than 60 years $^{[1]}$. Remarkably, $9 \%$ of heterosexual couples complain of infertility ${ }^{[2]}$. Approximately half of them affected are males, where azoospermia is the severest form ${ }^{[3]}$. Currently, testicular sperm extraction followed by Intracytoplasmic Sperm Injection (TESE-ICSI) is the only route to genetically father a child in couples with non-obstructive azoospermia $(\mathrm{NOA})^{[4]}$. This therapeutic modality has a cumulative chance of $25 \%$ of resulting in a live birth. Spermatozoa are successfully retrieved in $50 \%$ of men with NOA, and the subsequent use of these spermatozoa in one or more ICSI treatments results in a live birth rate of $50 \%{ }^{[4]}$. Currently, two methods are used to recover testicular sperms, random biopsy testicular sperm extraction (TESE) and microdissection TESE (micro-TESE). The procedure of micro-TESE performed with an operative microscope is generally considered to be the best method with higher spermatozoa recovery rates and minimal tissue loss in patients with $\mathrm{NOA}^{[5,6]}$. Repeated TESE may be needed after failed ICSI cycles with fresh and cryopreserved testicular 
sperms or following an unsuccessful attempt to retrieve testicular sperms. The role of repeated testicular biopsies to retrieve sperms in azoospermic men was assessed by several studies ${ }^{[7,8]}$. Sperm recovery rate in repeated TESE was reported as between 74.7 and $96 \%$ when the previous recovery procedure was successful ${ }^{[7]}$.

However, testicular spermatozoa were found only in $11.1-33 \%$ of azoospermic men with a previous spermnegative procedure ${ }^{[7]}$. We aimed in this study to identify the effect of the easiness of sperm retrieval - based on the difficulty of micro-TESE procedure, number of spermatozoa retrieved, and the number of surgical attempts - on clinical pregnancy following ICSI.

\section{PATIENTS AND METHODS}

\section{Study design and settings}

This was a retrospective study conducted from 2014 to 2019 and included 643 patients who underwent 712 Intracytoplasmic Sperm Injection (ICSI) cycles at a specialized in-vitro fertilization (IVF) center (Adam International Hospital, Giza, Egypt). We classified the patients into easy NOA (445) and difficult NOA (267) based on the difficulty of micro-TESE procedure, number of spermatozoa retrieved, and the number of surgical attempts. The patients signed written informed consent regarding the outcome and the risk associated with microTESE. Moreover, they were assured that confidentiality would be maintained during and after the study and information given would be used only for research purpose. Approval of the Ethical Committee was obtained and the study conforms to Helsinki declaration (1964).

\section{Inclusion criteria}

All the recruited participants experienced primary infertility of any duration and repeatedly azoospermic ejaculate with alkaline $\mathrm{pH}$ and with high or normal serum follicle stimulating hormone (FSH). We repeated micro-TESE at least after 6 months from the last attempt $t^{[9]}$.

\section{Exclusion criteria}

All participants with obstructive azoospermia, hypogonadotrophic hypogonadism, bilateral cryptorchidism, azoospermic factor microdeletion a or b, or abnormal karyotyping that contraindicates ICSI were excluded from the study. Moreover, patients' wives who produced less than three oocytes in response to controlled ovarian hyperstimulation were excluded from the study. Moreover, patients' wives who had anti-Müllerian hormone less than $0.7 \mathrm{ng} / \mathrm{ml}$ were excluded from the study as they had significantly lower fecundability compared with their counterparts with higher anti-Müllerian hormone levels ${ }^{[10]}$.

All the participants were subjected to the following: full clinical evaluation (history and examination) was done. At least two semen analyses with centrifugation and pellet analysis were conducted for each patient.
Basal hormonal profile (follicular stimulating hormone, luteinizing hormone, total testosterone, prolactin, and estradiol) was measured for the patients. All the patients with NOA had their serum hormone levels measured using chemiluminescence immunoassay (CLIA) technique, with values in the range of $1.5-14 \mathrm{mIU} / \mathrm{ml}$ for $\mathrm{FSH}$, $1.5-8 \mathrm{mIU} / \mathrm{ml}$ for Luteinizing hormone, $2.5-17 \mathrm{ng} / \mathrm{ml}$ for prolactin, $2.4-8.3 \mathrm{ng} / \mathrm{ml}$ for total testosterone, and $20-47 \mathrm{pg} / \mathrm{ml}$ for estradiol taken as normal. Early follicular phase FSH was measured for their wives who provided a blood sample on either day 2, 3, or 4 of the menstrual cycle and then the serum was stored frozen at $-80^{\circ} \mathrm{C}$ until analyses where FSH was measured by a direct immunochemiluminometric assay using the automated Immulite system (Siemens Medical Solutions Diagnostics, Malvern, Pennsylvania, USA) (3-51 $\mathrm{mIU} / \mathrm{ml})^{[11,12]}$. Peripheral blood karyotyping and azoospermic factor microdeletion by PCR were offered for each patient with NOA.

Evaluation of prior histopathology reports and/or slides for patients who underwent previous micro-TESE was done. Testicular spermatozoa retrieval for each patient was done using micro-TESE technique ${ }^{[5,6]}$. Notably, we determined the total numbers of retrieved, injected (M2), and fertilized oocytes; numbers and quality of embryos formed; chemical pregnancy; clinical pregnancy; and miscarriage for their wives. Two protocols were used for controlled ovarian hyperstimulation. In brief, the long GnRH agonist protocol was based on the administration of daily leuprorelin (Lucrin; Abbvie, North Chicago, Illinois, USA) or triptorelin (Decapeptyl; Ferring, Saint-Prex, Switzerland) in the mid-luteal phase before the stimulation cycle. The administration of recombinant FSH (Gonal F; Merck, Kenilworth, NJ, USA), highly purified hMG (Menopur; Ferring Saint-Prex, Switzerland), or urinary FSH (Fostimon; IBSA, Lugano, Switzerland) was started when pituitary desensitization was achieved (7-10 days after the initiation of GnRH agonists), as evidenced by the absence of ovarian follicles more than $10 \mathrm{~mm}$, endometrial thickness less than $4 \mathrm{~mm}$ on transvaginal ultrasound examination, and serum E2 less than 30. Alternatively, in the GnRH antagonist protocol, Cetrorelix (Cetrotide; Merck, Kenilworth, NJ, USA) was started daily $0.25 \mathrm{mg} /$ days subcutaneously on day 5 or 6 of stimulation. We used transvaginal ultrasound with a frequency of $7 \mathrm{MHz}$ (Medison Digital Color MT; Medison Co. Ltd, Seoul, Korea). When two or more follicles reached a diameter of $18 \mathrm{~mm}$, human chorionic gonadotrophins (Choriomon, 10000 IU, intramuscular; IBSA, Lugano, Switzerland)) were administered $36 \mathrm{~h}$ before recovery of large ovarian follicles by transvaginal needle-guided ultrasound aspiration. Modified Ham's F-10 with HEPES (99168; Irvine Scientific, Santa Ana, California, USA) was used to flush the ovarian follicles in case of difficult retrieval or if few follicles were seen.

The ICSI procedure was performed after the preparation of the oocytes according to the guidelines 
of Palermo et al. ${ }^{[13] .}$ The injected ova (metaphase II oocytes) were incubated for fertilization and further cleavage. Fertilization was confirmed after $16-18 \mathrm{~h}$ by the observation of two distinct pronuclei (2PN) and two polar bodies. Embryo quality was assessed on days 2 or 3 after oocyte retrieval using a four-point score as described by Racowsky et al. ${ }^{[14]}$.The score depended on cell number, as 4 cell stage on day 2 and 8 cell stage on day 3 having the highest score, and additional points were added for each of the following features : Absence of fragmentation or fragmentation involving less than $25 \%$ of embryonic surface and absence of irregularities in blastomere size or shape ${ }^{[14]}$. Immature, deformed and postmature oocytes were excluded. Blastocyst grading was done according to Gardner et al. ${ }^{[15]}$. Thus, embryos with the best morphology and the most advanced stage of development were selected and transferred, by the same clinician, using a K-Jet embryo transfer catheter (Cook Echo-Tip catheter, Brisbane, Queensland, Australia). All the female patients received luteal supplementation by intravaginal administration of $400 \mathrm{mg}$ natural-micronized progesterone twice daily (Prontogest; Marcyrl, El Obour City, Egypt. Chemical pregnancy was identified as a positive $\beta$-human chorionic gonadotrophin assay,whereas clinical pregnancy was identified by the presence of at least one gestational sac with fetal heart beat detected by transvaginal ultrasound examination, and miscarriage was identified as pregnancy loss before 24 weeks of gestation ${ }^{[16]}$.

\section{Statistical analysis}

We used SPSS version 26 (IBM SPSS Statistics for Windows, Armonk, NY: IBM Corp) in the analysis.
Descriptive data presented as mean $\pm \mathrm{SD}$, frequencies, and percentages. We used the independent Student t-test to compare between easy and difficult NOA. Contingency table analysis was used to compare ICSI outcome using $\chi^{2}$ or Fisher's exact test as appropriate. Potential clinical predictors including difficulty of micro-TESE were correlated with the clinical pregnancy rate using Pearson correlation. Only significant predictors in the correlation were used as independent variables in the regression analysis. $P$ value less than 0.05 is considered to be significant, with all values at two-tailed until otherwise stated.

\section{RESULTS}

The retrospective analysis included 643 men with azoospermia who underwent 712 TESE/ICSI attempts. The mean age of males was $37.4 \pm 7$ years, whereas it was $28.4 \pm 4.5$ for the wives. The patients' demographics, the clinical characteristics, and the ICSI findings are shown in Table 1. We classified the ICSI attempt according to the difficulty of surgical testicular spermatozoa retrieval, number of spermatozoa harvested, and the repetition of TESE attempts into easy(445) and difficult (267) NOA, as shown in Fig.1. Testicular histopathology was similar in both groups. Sertoli cell-only (SCO) picture prevailed in both groups (35 and 37\% in easy and difficult NOA, respectively), and hypospermatogenesis was the second most common finding (27 and $21 \%$ in easy and difficult NOA, respectively). Detailed histopathological pictures are shown in Table 2.

Table 1: Patients demographics and characteristics

\begin{tabular}{lcc}
\hline & Mean & SD \\
\hline Male age (Y) & 37.4 & 7.0 \\
Female age (Y) & 28.4 & 4.5 \\
Male FSH (mIU/ml) & 17.88 & 14.86 \\
Female FSH (mIU/ml) & 7.05 & 2.3 \\
Cummulus Mass & 16.1 & 8.6 \\
Mature oocytes & 12.5 & 6.9 \\
Injected oocytes & 12.8 & 7.2 \\
Fertilized & 8.6 & 5.6 \\
Fertilization Rate (\%) & 67.7 & 20.2 \\
Embryos & 8.5 & 5.5 \\
Blastocyst in Day 5 & 4.1 & 3.1 \\
Total Blastocyst & 4.5 & 3.5 \\
Embryo Transfer Day & 2.8 & 1.9 \\
Embryo Transfer Number & 2.3 & 0.73 \\
Good Embryos Transferred & 1.2 & 1.0 \\
Fair Embryos Transferred & 0.8 & 1.7 \\
Bad Embryos Transferred & 0.6 & 0.9 \\
\hline
\end{tabular}

FSH, Follicle Stimulating Hormone. 


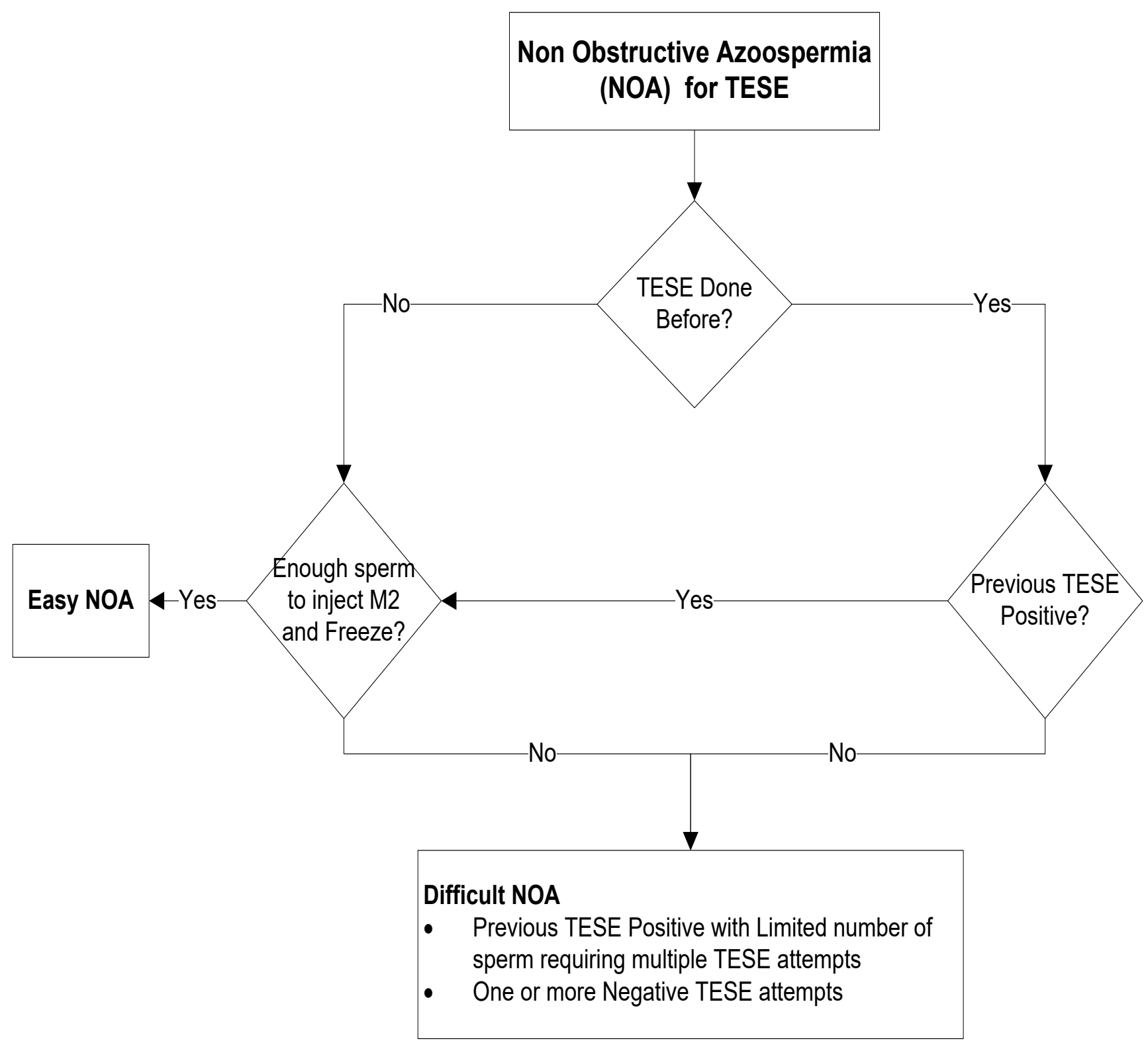

NOA=Non Obstructive Azoospermia, TESE $=$ Testicular Sperm Extraction

Fig. 1: Proposed criteria of easiness of sperms retrieval in Non-Obstructive Azoospermia. 
Table 2: Basic differences between easy non-obstructive azoospermia (NOA) and difficult NOA

\begin{tabular}{|c|c|c|c|}
\hline & Easy NOA & Difficult NOA & $P$ \\
\hline \multicolumn{4}{|l|}{ TESE Classification: } \\
\hline Previous positive TESE & $146(32.8 \%)$ & & \\
\hline 1st Time TESE & $299(67.2 \%)$ & & \\
\hline One Previous negative TESE & & $119(44.6 \%)$ & \\
\hline Two Previous negative TESE & & $48(18.0 \%)$ & \\
\hline Third Time TESE or more & & $100(37.5 \%)$ & \\
\hline Right testicular size & & & 0.174 \\
\hline Small & $123(27.6 \%)$ & $72(27.0 \%)$ & \\
\hline Moderate & $220(49.4 \%)$ & $148(55.1 \%)$ & \\
\hline Normal & $102(22.9 \%)$ & $47(17.6 \%)$ & \\
\hline Left testicular size & & & 0.113 \\
\hline Small & $121(27.2 \%)$ & $85(31.5 \%)$ & \\
\hline Moderate & $216(48.5 \%)$ & $133(49.8 \%)$ & \\
\hline Normal & $108(24.3 \%)$ & $48(18.0 \%)$ & \\
\hline Testicular Pathology & & & 0.228 \\
\hline Tubular Sclerosis & $4(0.9 \%)$ & $3(1.1 \%)$ & \\
\hline Sertoli Cell Only & $154(34.8 \%)$ & $97(36.6 \%)$ & \\
\hline Primary spermatocyte arrest & $87(19.6 \%)$ & $63(23.8 \%)$ & \\
\hline Spermatid arrest & $50(11.3 \%)$ & $22(8.3 \%)$ & \\
\hline Mixed arrest & $30(6.8 \%)$ & $25(9.4 \%)$ & \\
\hline Hypospermatogenesis & $118(26.6 \%)$ & $55(20.8 \%)$ & \\
\hline Round Spermatids identified & & & 0.062 \\
\hline No & $245(55.3 \%)$ & $163(61.5 \%)$ & \\
\hline Yes & $198(44.7 \%)$ & $102(38.5 \%)$ & \\
\hline Female diagnosis & & & 0.411 \\
\hline No medical Issues & $381(95.3 \%)$ & $203(94.4 \%)$ & \\
\hline Polycystic ovaries & $15(3.8 \%)$ & $10(4.7 \%)$ & \\
\hline Polyp & $3(0.8 \%)$ & 0 & \\
\hline Uterine anomaly & $1(0.3 \%)$ & $1(0.5 \%)$ & \\
\hline Ovarian cyst & 0 & $1(0.5 \%)$ & \\
\hline Stimulation Protocol & & & 0.425 \\
\hline Fixed Antagonist & $420(94.4 \%)$ & $248(93.0 \%)$ & \\
\hline Long & $25(5.6 \%)$ & $19(7.0 \%)$ & \\
\hline Sperm Used & & & 0.436 \\
\hline Fresh Sperm & $242(54.4 \%)$ & $154(57.7 \%)$ & \\
\hline Frozen Sperm & $203(45.6 \%)$ & $113(42.3 \%)$ & \\
\hline \multicolumn{4}{|l|}{ ICSI Outcome } \\
\hline Fertilization Rate & $3973 / 5938(66.9 \%)$ & $2107 / 3103(67.9 \%)$ & 0.345 \\
\hline Implantation Rate & $215 / 729(29.5 \%)$ & $88 / 446(19.7 \%)$ & $0.0002^{*}$ \\
\hline Clinical pregnancy & $167 / 299(55.8 \%)$ & $68 / 194(25.1 \%)$ & $0.0001^{*}$ \\
\hline
\end{tabular}

ICSI, Intracytoplasmic Sperm Injection; NOA, Non-Obstructive Azoospermia; TESE, Testicular Sperm Extraction. *Statistically significant. 
The mean age of the females and the mean FSH levels were significantly lower in the easy NOA group compared with the difficult group $(27.5 \pm 4.3$ vs $29.9 \pm 4.6$ years, $P=0.0001$, and $6.8 \pm 2.1$ vs $7.4 \pm 2.5 \mathrm{mIU} / \mathrm{ml}, P=0.004$, respectively). No significant differences were observed between the 2 groups regarding the mean male age, FSH level, and testicular size (Table 3). The mean cumulus mass, mature oocytes harvested and injected, the number of fertilized eggs, number of embryos, number of blastocysts in day 5 , and the total number of blastocyst developed were significantly higher in the easy NOA as compared with the difficult group (Table 3). The fertilization rate was almost equal in both groups [3973/5938 (66.9\%) in easy NOA compared with $2107 / 3103(67.9 \%)$ in the difficult group, $P=0.345]$. However, the implantation rate and the clinical pregnancy rate were significantly higher in the easy group as compared with the difficult group [215/729 (29.5\%) vs $88 / 446(19.7 \%)$, respectively, $P=0.0002$, and $167 / 299$ (55.8\%) vs 68/194 (25.1\%), respectively, $P=0.0001]$ (Table 2 and Fig.2). Female age, serum FSH levels, the severity of NOA, cumulus mass, and M2 injected correlated significantly with the clinical pregnancy. On the contrary, male age, FSH levels, testicular histopathology, and sperm used (fresh vs frozen) did not correlate with the clinical pregnancy (Table 4). Female age, serum FSH level, and the severity of NOA were used as early independent factors that could predict clinical pregnancy after ICSI. We excluded the number of mature oocytes injected from the regression because it is a late predictor. The regression analysis showed that the severity of NOA is the only predictor of clinical pregnancy (odds ratio $=1.70,95 \%$ confidence interval $=1.1-2.71, P=0.025)$. The model showed that female age, early follicular FSH level, and cumulus mass could not predict ICSI outcome in those patients.

Table 3: Compares between easy and difficult Non Obstructive Azoospermia (NOA) in terms of clinical parameters and ICSI outcome.

\begin{tabular}{|c|c|c|c|c|c|c|c|}
\hline & \multicolumn{3}{|c|}{ Easy NOA (445) } & \multicolumn{3}{|c|}{ Difficult NOA (267) } & \multirow{2}{*}{$P$} \\
\hline & $\mathrm{N}$ & Mean & SD & $\mathrm{N}$ & Mean & $\mathrm{SD}$ & \\
\hline Male age $(Y)$ & 445 & 37.0 & 7.2 & 267 & 38.0 & 6.5 & 0.060 \\
\hline Female age (Y) & 445 & 27.5 & 4.3 & 267 & 29.9 & 4.6 & $0.0001 *$ \\
\hline Male FSH (mIU/ml) & 441 & 17.4 & 13.3 & 264 & 18.6 & 17.2 & 0.336 \\
\hline Female FSH (mIU/ml) & 445 & 6.8 & 2.1 & 215 & 7.4 & 2.5 & $0.004 *$ \\
\hline Cummulus mass & 399 & 16.9 & 8.8 & 257 & 14.7 & 8.3 & $0.001 *$ \\
\hline Mature Oocytes (M2) & 399 & 13.1 & 7.0 & 257 & 11.4 & 6.7 & $0.003 *$ \\
\hline M2 Injected & 442 & 13.4 & 7.1 & 257 & 11.6 & 7.4 & $0.001 *$ \\
\hline Fertilized & 442 & 9.0 & 5.5 & 267 & 7.9 & 5.6 & $0.011^{*}$ \\
\hline Fertilization Rate & 442 & 67.3 & 19.6 & 266 & 68.3 & 21.2 & 0.513 \\
\hline Embryos & 442 & 8.9 & 5.4 & 266 & 7.8 & 5.6 & $0.011^{*}$ \\
\hline Blastocyst D5 & 147 & 4.3 & 3.1 & 38 & 3.1 & 2.9 & $0.025^{*}$ \\
\hline Total Blastocyst & 261 & 4.7 & 4.2 & 109 & 4.2 & 3.5 & 0.221 \\
\hline Embryo transfer Number & 306 & 2.4 & 0.7 & 198 & 2.2 & 0.7 & 0.051 \\
\hline Good Embryos transferred & 290 & 1.3 & 1.0 & 191 & 1.0 & 1.0 & 0.170 \\
\hline Fair Embryos transferred & 240 & 0.8 & 2.0 & 187 & 0.6 & 0.8 & 0.071 \\
\hline Bad Embryos transferred & 231 & 0.4 & 1.0 & 184 & 0.4 & 0.9 & 0.954 \\
\hline
\end{tabular}

FSH, Follicle Stimulating Hormone; NOA, Non-Obstructive Azoospermia.

*Statistically significant. 


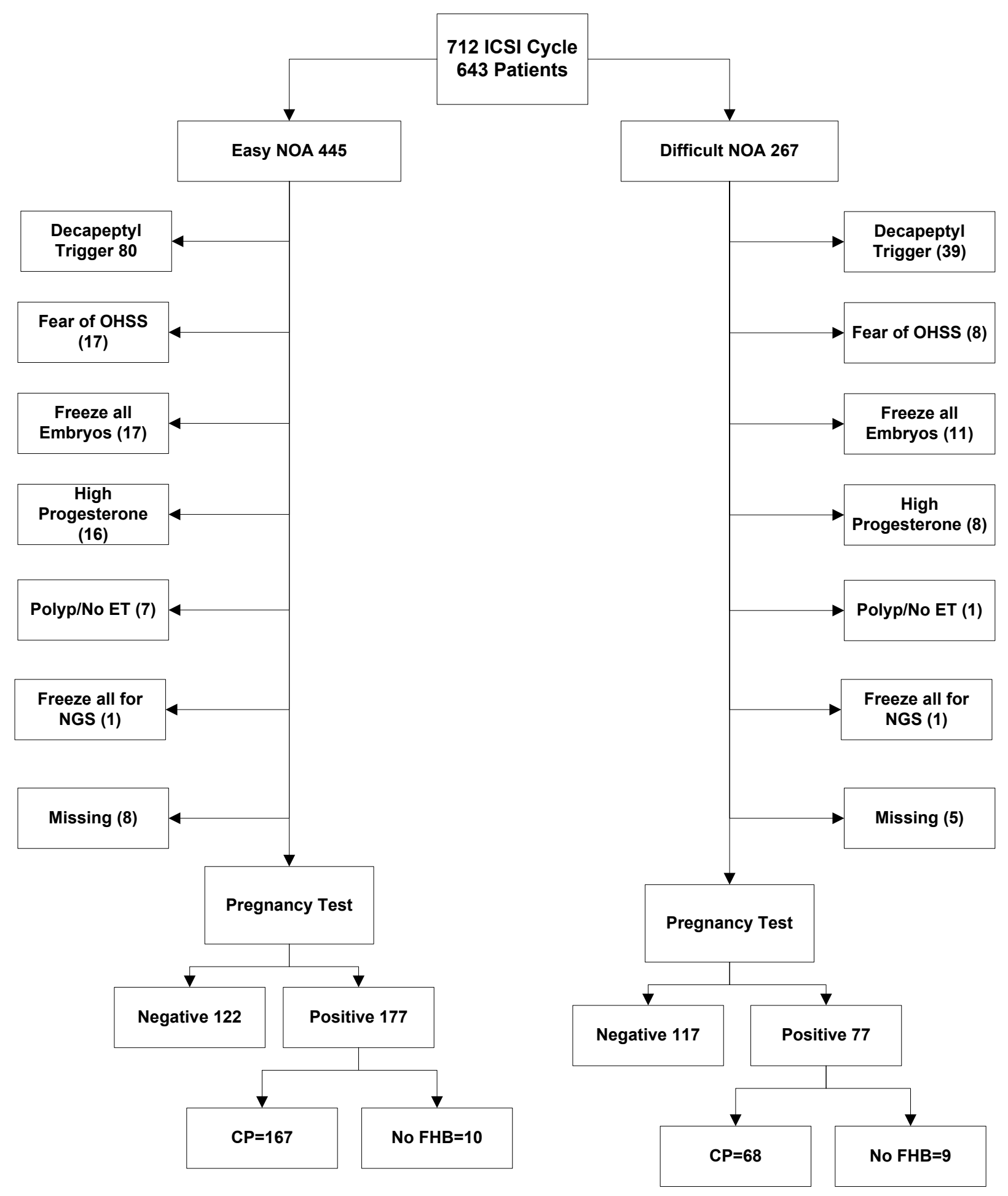

$\mathrm{CP}=$ Clinical Pregnancy, ET $=$ Embryo Transfer, FHB $=$ Fetal Heart Beat, ICSI $=$ Intracytoplasmic Sperm Injection,NOA= Non Obstructive Azoospermia, NGS=Next Generation Sequencing, OHSS= Ovarian Hyperstimulation Syndrome,

Fig. 2: ICSI cycles in Non-obstructive Azoospermia (Easy versus Difficult). 
Table 4: Correlation between the clinical pregnancy and male and female clinical parameters

\begin{tabular}{lcc}
\hline & \multicolumn{2}{c}{ Clinical Pregnancy } \\
\cline { 2 - 3 } & $r$ & $P$ \\
\hline Male Age & -.069 & 0.138 \\
Female Age & -0.136 & $0.003^{*}$ \\
Male FSH & -0.014 & 0.758 \\
Female FSH & -0.11 & $0.05^{*}$ \\
NOA (easy vs. Difficult) & -0.205 & $0.0001^{*}$ \\
Testicular Pathology & -0.073 & 0.111 \\
Spermatid identification & -0.062 & 0.182 \\
Female Diagnosis & -0.059 & 0.239 \\
Sperm Used (fresh vs. frozen) & 0.059 & 0.23 \\
Cumulus Mass & 0.122 & $0.012^{*}$ \\
MII Injected & 0.145 & $0.003^{*}$ \\
\hline
\end{tabular}

FSH, Follicle Stimulating Hormone; MII, Metaphase II oocytes; NOA, Non-Obstructive Azoospermia.

*Statistically significant.

Table 5: Regression analysis of the potential clinical predictors of clinical pregnancy

\begin{tabular}{lccccc}
\hline & & & \multicolumn{2}{c}{$95 \%$ CI for Exp(B) } \\
\hline Female age & $\mathrm{B}$ & Significance & OR & Lower & Upper \\
FemaleFSH & -0.040 & 0.142 & 0.960 & 0.910 & 1.014 \\
Non Obstructive Azoospermia & -0.062 & 0.227 & 0.940 & 0.850 & 1.039 \\
Cummulus mass & 0.533 & 0.026 & 1.703 & 1.065 & 2.724 \\
Constant & 0.005 & 0.783 & 1.005 & 0.970 & 1.041 \\
\hline
\end{tabular}

CI, Confidence Interval; FSH, Follicle Stimulating Hormone; NOA, Non-Obstructive Azoospermia (Easy vs Difficult); OR, Odds Ratio.

\section{DISCUSSION}

Our study had shown a significant correlation between female age and clinical pregnancy. This finding can be explained by the fact that woman's age imposed a significant footprint on early embryo morphological development (3 Pronuclei), as compromised meiotic spindle function occurs with increasing female age ${ }^{[17]}$. Similarly, van Loendersloot et al. ${ }^{[18]}$ stated that female age can be considered the most powerful predictive factor for pregnancy chances in IVF. Additionally, Bocca et al. ${ }^{[19]}$ had demonstrated the significance of female age on IVF and ICSI outcomes. However, it should be noted that Bocca et al. ${ }^{[19]}$ had conducted their study on patients with obstructive OA and those with NOA and found significantly high fertilization rates in patients with OA than patients with NOA whose partners were younger than 35 years old. Meanwhile, our study did not show 
any correlation between male age and clinical pregnancy. In agreement with our finding, $\mathrm{Wu}$ et al. ${ }^{[20]}$ reported no correlation between paternal age and clinical pregnancy rate. In contrast, Shrem et al. ${ }^{[21]}$ had shown that paternal age above 37 years was significantly adversely correlated with clinical pregnancy. Besides, serum FSH levels of the females in the easy NOA group were significantly lower and had shown a significant correlation with clinical pregnancy than those in the difficult NOA group which could be explained by the fact that patients with elevated FSH levels are associated with decline in the quantity of the ovarian reserve with subsequent reduced pregnancy and live birth rates ${ }^{[22]}$. Moreover, early follicular phase FSH has been assessed as a marker of natural fertility, especially among subfertile women ${ }^{[23]}$.

Furthermore, our study revealed a significant association between the maturity of the oocytes punctured, mean number of fertilized oocytes, quality of the embryos formed on day 3, blastocysts formed on day 5 , and gestational sacs determined by transvaginal ultrasound and favorable ICSI. In the same context, Lazzaroni-Tealdi et al. ${ }^{[24]}$ had revealed that oocyte size demonstrated significant effect on embryo quality and was an independent predictor of embryo quality after adjustment for age. Consistently, Yin et al. ${ }^{[25]}$ had shown that transfer of equal number of embryos on day 3 was significantly associated with the highest clinical pregnancy rates in women younger than 35 years old. On the contrary, Capalbo et al. ${ }^{[26]}$ had demonstrated that the well-settled parameters of blastocyst evaluation were not reliable enough as indicators to improve the selection among euploid embryos. Thus, all poor morphology and slower growing expanded blastocysts should be biopsied and similarly considered for frozen embryo transfer cycles ${ }^{[26]}$. Moreover, neither testicular histopathology nor FSH level in patients with NOA demonstrated correlation with clinical pregnancy. In the same line, a study conducted by Guler et al. ${ }^{[27]}$ stated that testicular histopathology did not influence pregnancy rate in patients with NOA. Furthermore, Shrem et al. ${ }^{[21]}$ did not find any correlation between male FSH level and clinical pregnancy. On the contrary, a study conducted by Zitzmann et al. ${ }^{[28]}$ had shown that an FSH cutoff of 20 IU predicted pregnancy. Moreover, our study did not demonstrate any correlation between fresh or frozen spermatozoa and clinical pregnancy. Similarly, a recent systematic review and metaanalysis concluded that ICSI outcome is not affected by whether the retrieved testicular sperm is fresh or frozen ${ }^{[29]}$.

Interestingly, our study is one of the first to demonstrate the crucial role of the easiness of getting spermatozoa from patients with NOA whose partners are normal to achieve clinical pregnancy with respect to the difficulty of TESE procedure, number of spermatozoa retrieved, and the number of surgical attempts. Thus, easy NOA cases were considered the only independent predictor of clinical pregnancy after adjustment of the other factors.
Consistently, a study stressed on the essential need of developing new treatment options to retrieve spermatozoa from NOA cases to enable biological fatherhood ${ }^{[30]}$. Admittedly, the retrospective nature and the relatively small sample can be regarded as the major limitations of this study. Moreover, inability to determine the live birth rate can be considered another limitation.

\section{CONCLUSION}

In brief, easy testicular sperm retrieval is an independent factor that is associated with positive ICSI outcomes in couples with NOA.

\section{CONFLICT OF INTEREST}

There are no conflicts of interest.

\section{REFERENCES}

1. WHO and World Bank World Report on Disability 2011. Available at:http://www.who.int/disabilities/ world report/2011/en/ (Accessed September 2015).

2. Boivin J, Bunting L, Collins J A, Nygren K G. International estimates of infertility prevalence and treatment-seeking: potential need and demand for infertility medical care. Hum Reprod 2007; 22:1506-1512.

3. Agarwal A, Mulgund A, Hamada A, Chyatte M R. A unique view on male infertility around the globe. Reprod Biol Endocrinol2015; 26:13-37.

4. Dabaja A A, Schlegel P N. Microdissection testicular sperm extraction: an update. Asian $\mathrm{J}$ Androl $2013 ; 15: 35-39$.

5. Schlegel P N. Testicular sperm extraction: microdissection improves sperm yield with minimal tissue excision. Hum Reprod 1999; 14:131-135.

6. Amer M, Ateyah A, Hany R, Zohdy W. Prospective comparative study between microsurgical and conventional testicular sperm extraction in non-obstructive azoospermia: follow-up by serial ultrasound examinations. Hum Reprod 2000; 15:653-656.

7. Vernaeve $\mathrm{V}$, Verheyen $\mathrm{G}$, Goossens A, VanSteirteghem A, Devroey P, Tournaye H. How successful is repeat testicular sperm extractionin patients with azoospermia. Hum Reprod2006; 2006:1551-1554.

8. Haimov Kochman R, Lossos F, Nefesh I, Zentner 
B S, Moz Y, Prus D, et al. The value of repeat testicular sperm retrieval in azoospermic men. Fertil Steril2009; 91 (4Suppl):14013.

9. Amer M, Haggar S E, Moustafa T, Abd El-Naser T, Zohdy W. Testicular sperm extraction: impact of testicular histology on outcome, number of biopsies to be performed and optimal time for repetition. Hum Reprod1999; 14:3030-3034.

10. Steiner A Z, Herring A H, Kesner J S, Meadows J W, Stanczyk F Z, Hoberman S, Baird D D. Antimullerian hormone as a predictor of natural fecundability in women aged 30-42 years. Obstet Gynecol 2011; 117:798-804.

11. Muasher S J, Oehninger S, Simonetti S, Matta J, Ellis L M, LiuH-C, et al.The value of basal and/or stimulated serum gonadotropin levels in prediction of stimulation response and in vitro fertilization outcome. Fertil Steril1988; 50:298-307.

12. Hansen L. M, Batzer F R, Gutmann J N, Corson S L, Kelley M P, Gocial B. Evaluating ovarian reserve: follicle stimulating hormone and oestradiol variability during cycle days $2-5$. Hum Reprod 1996; 3:486-489.

13. Palermo G, Joris H, Devroey P, Van Steirteghem A C. Pregnancies after intra cytoplasmic injection of single spermatozoon into an oocyte. Lancet 1992; 340:17-18.

14. Racowsky C, Vernon M, Mayer J, Ball G D, Behr B, Pomeroy K O, et al. Standardization of grading embryo morphology. Fertil Steril 2010; 94:1152-1153.

15. Gardner D K, Stevens J, Sheehan C B, Schoolcraft W B. Morphological assessment of the human blastocyst. In:Elder K T, Cohen J, editors. Analysis of the Human Embryo. London: Taylor \& Francis; 2007.79-87.

16. Antinori M, Licata E, Dani G, Cerusico C, Versaci C, D'AngeloD, et al. Intra cytoplasmic morphologically selected sperm injection: a prospective randomized trial. Reprod Biomed Online2008; 16:835-841.

17. Grøndahl M L, Christiansen S L, Kesmodel U S, Agerholm I E, Lemmen J G, Lundstrøm $\mathrm{P}$, et al. Effect of women'sage on embryo morphology, cleavage rate and competence - a multicenter cohort study. PLoS One2017; 12:e172456.

18. van Loendersloot L L, van Wely M, Limpens
J, Bossuyt P M, Repping S, van der Veen F. Predictive factors in in vitro fertilization (IVF): a systematic review and metaanalysis. Hum Reprod Update 2010; 16:577-589.

19. Bocca S, Moussavi V, Brugh V, Morshedi M, Stadtmauer L,Oehninger S.ICSI outcomes in men undergoing TESE for azoospermia and impact of maternal age. Andrologia 2017; 49:e12617.

20. Wu Y, Kang X, Zheng H, Liu H, Huang Q, Liu J. Effect of paternal age on reproductive outcomes of intracytoplasmic sperm injection. PLoS One 2016; $11: \mathrm{e} 0149867$.

21. Shrem G, Brudner Y, Atzmon Y, Michaeli M, Ellenbogen A, Shalom-Paz E. The influence of obesity, smoking, and serum follicular stimulating hormone in azoospermic patients on testicular sperm extraction-intra cytoplasmic sperm injection outcomes: a retrospective cohort study. Medicine 2019; 98:4.

22. AbdallaH, ThumMY. An elevated basal FSH reflects a quantitative rather than qualitative decline of the ovarian reserve. Hum Reprod2004; 19:893-898.

23. 23. van der Steeg J W, Steures P, Eijkemans M J, Habbema J D, Hompes P G, Broekmans F J, et al. Predictive value and clinical impact of Basal follicle-stimulating hormone in subfertile, ovulatory women. J Clin Endocrinol Metab 2007; 92:2163-2168.

24. Lazzaroni-Tealdi E, Barad D H, Albertini D F, Yu Y, Kushnir V A, RussellH, et al. Oocyte scoring enhances embryo-scoring in predicting pregnancy chances with IVF where it counts most. PLoS One2015; 10:1-13.

25. Yin H, Jiang H, He R, Wang C, Zhu J, Luan K. The effects of fertilization mode, embryo morphology at day 3 , and female age on blastocyst formation and the clinical outcomes. Syst Biol Reprod Med2015; 61:50-56.

26. Capalbo A, Rienzi L, Cimadomo D, Maggiulli $\mathrm{R}$, Elliott T, Wright $\mathrm{G}$, et al. Correlation between standard blastocyst morphology, euploidy and implantation: an observational study in two centers involving 956 screened blastocysts. Hum Reprod2014; 29:1173-1181.

27. Guler I, ErdemM, ErdemA, DemirdağE, TuncL Bozkurt N, et al. Impact of testicular histopathology as a predictor of sperm retrieval and pregnancy 
outcome in patients with nonobstructive azoospermia: correlation with clinical and hormonal factors. Andrologia 2016; 48:765-773.

28. Zitzmann M, Nordhoff V, von Schönfeld V, Nordsiek-Mengede A, Kliesch S, Schuring A N, et al. Elevated follicle stimulating hormone levels and the chances for azoospermic men to become fathers after retrieval of elongated spermatids from cryopreserved testicular tissue. Fertil Steril2006; 86:339-347.
29. Yu Z, Wei $Z$, Yang J, Wang $T$, Jiang $H$, $\mathrm{Li} \mathrm{H}$, et al. Comparison of intracytoplasmic sperm injection outcome with fresh versus frozen-thawed testicular sperm in men with nonobstructive azoospermia: a systematic review and meta-analysis. J Assist Reprod Genet 2018; 35:1247-1257.

30. Klami R, Mankonen H, Perheentupa A. Microdissection testicular sperm extraction in finland - results of the first 100 patients. Acta Obstet Gynecol Scand2018; 97:53-58. 